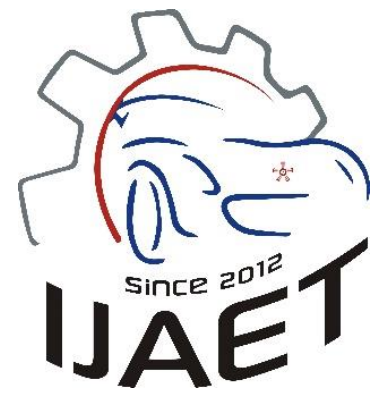

e-ISSN: 2146 - 9067

International Journal of Automotive

Engineering and Technologies

journal homepage:

https://dergipark.org.tr/en/pub/ijaet

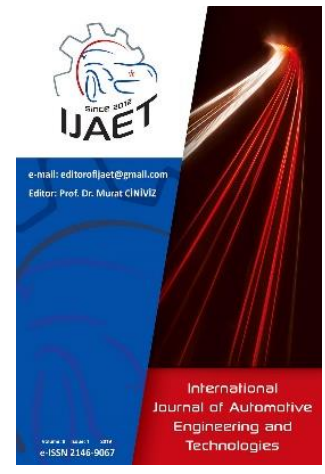

Original Research Article

\title{
Investigation on hydrodynamic characteristics of a Stirling regenerator matrix using porous media approach: a CFD study
}

1 * Gazi University, Technology Faculty, Automotive Engineering Department, Teknikokullar, 06560, Ankara, Turkey

\begin{abstract}
ARTICLE INFO
$10000-0002-8862-7662$

* Corresponding author duyguipci@gazi.edu.tr

Doi: 10.18245/ijaet.779636

Received: Agu 18, 2020

Accepted: Oct 20, 2020

Published by Editorial Board Members of IJAET

(C) This article is distributed by Turk Journal Park System under the CC 4.0 terms and conditions.
\end{abstract}

\begin{abstract}
In this study, hydrodynamic characteristics of a Stirling regenerator matrix are predicted by porous medium based modeling. A regenerator is designed to be used in the beta type Stirling engine. CFD analysis of the designed regenerator is performed by the ANSYS Fluent software with porous media model. The flow properties in porous media are generally approximated by Forchheimer or Ergun flow regime. The equation of the Forchheimer flow model consists of two-term: viscous loss and the inertial loss. The viscous resistance and inertial resistance factors of the porous medium to be used in CFD analysis is determined from published experimental results for a regenerator made by stainless steel with the porosity of $70 \%$. The CFD simulation are validated by comparing the calculated the velocity distributions at the exit of the regenerator with results of previously published paper. By using both resistance factors, pressure drops, and friction factors of the regenerator matrix are calculated via CFD analysis. The friction results are interpolated to generate a correlation equation that can be able to calculate the pressure drops in the flow direction of the regenerator and to use in future numerical simulations.
\end{abstract}

Keywords: Stirling engine regenerator, Porous media, Pressure drop, Friction factor

\section{Introduction}

The first regenerator idea is identified by Robert Stirling namely as an economizer in a patent that is known as the regenerative principle in 1816 [1]. The regenerator which consists of a packed stack of wire screens or a solid matrix structure is one of the crucial components in the Stirling engine. It is located between the heater and cooler of the engine and works as a thermal energy storage component in the engine. The regenerator stores the waste energy from the working fluid when the working fluid flows from the heater to cooler and transfers the heat to working fluid during the reverse process $[2$,
3]. Since the regenerator provides thermal energy equilibrium of the thermodynamic process in the closed cycle, the performance of the regenerator instantly affects the engine efficiency $[4,5,6]$. Numerically predicting the heat transfer and pressure drop phenomenon in regenerator are too complex due to its porous structure [7, 8]. To numerically examined flow and heat transfer phenomena in a regenerator, commercial or open-source Computational Fluid Dynamics (CFD) software packages such as Star CCM+, Fluent, CFX, Open Foam, and etc. exists. These software packages implement macroscopic porous media model that required 
porosity, permeability, viscous and inertial coefficients as the inputs [9]. To determine the input parameters, pressure drop, and temperature variations of porous media are needed to be obtained by the experiments.

Chen et al. conducted a study focused on examining the effects of various regenerator parameters on helium charge gamma-type twin power piston Stirling engine performance. The authors presented the results of experiments performed with the parameters of regenerator matrix material, matrix arrangement, matrix wire diameter, and fill factor versus engine speed at several engine's hot-end temperatures. As a result, they reported that very small matrix wire diameter reduces the engine effectiveness because of resulting in large pressure drop and also the perpendicular installation of matrix screen increases the engine performance [10]. Costa et al. carried out a numerical study to derive a heat transfer correlation equation for both types stacked and wound woven wire matrices of the porous medium forming the Stirling regenerator. Authors use the finite volume method (FVM) based numerical flow solver for the laminar flow solutions by discretizing the continuity and momentum equations via second order upwind scheme. To validate the numerical method, empirical correlation results obtained from experiments for a misaligned stacked woven wire matrix are compared with numerical results and found to be less than $6 \%$ deviation between them [11]. In another study by the authors with more coworkers, the pressure drop and heat transfer characteristics of Stirling engine regenerators are investigated under oscillating flow conditions by conducting on two stages study, experimental and numerical. In experiments, instantaneous pressure drop and temperature distributions in regenerator determined under oscillating flow conditions which is established with the operating conditions of a commercial micro-CHP Stirling engine WhisperGen. As a result, they obtained experimental friction factor and Stanton number correlations. In numerical study, authors generated wire matrix models based on the Stirling regenerator matrix of the WhisperGen ${ }^{\mathrm{TM}}$ micro-CHP Stirling. The numerical simulate of matrix models are executed with finite volume method (FVM) based numerical flow solver for different porosity levels. The authors reported that the numerical results are found to be good agreement with the results of Darcy's law and to validate the models, further examinations are required for wide parametric range [12]. Kim et al. examine and optimize the free piston Stirling regenerator performance with respect to regenerator diameter porosity, mesh screen structure and mesh diameter via SAGE program which solves theoretical and empirical Stirling cycle equations. For porosity level under $88 \%$, random woven screen generate more indicated work and efficiency than random wire screen and after the porosity level $88 \%$, reverse situation is observed. The authors reported that large and small fiber diameter dimensions decrease the regenerator efficiency for same porosity level. The dead volume of regenerator increase with expanding the regenerator volume, thus, indicated work and efficiency of the regenerator decrease because of decreasing in heat transfer between working gas and matrix structure [13]. Mohammadi and Jafarian carry out a CFD study to investigate the hydrodynamic characteristic of commercial beta type Stirling engine namely GPU-3 under oscillating flow condition. The authors proposed a CFD model by neglecting mechanical friction, shuttle effect, mass leakage and regenerator ineffectiveness. The simulation of the Stirling engine is performed with OpenFoam fluent solver via dynamic mesh and $\mathrm{k}-\omega$ SST turbulence model. To validate the CFD model, second order thermodynamic theoretical model are developed. Authors are compared CFD results with results of theoretical model and experiments and found to be good agreement with simulation results [14]. Gedeon and Wood conducted on a study experimentally to investigate effect of the regenerator matrix type on the performance of Stirling engine. Authors obtained the correlation equations of friction factor and Nusselt number for matrix types of wire screen mesh and felt by using different mesh densities [15].

In this paper, the hydrodynamic characteristic of a beta type Stirling engine regenerator examined based on a porous media model by ANSYS Fluent program. The viscous and inertial resistance factors are determined from published experimental [16] data for the wire screen mesh model of the regenerator. For the 
proposed regenerator, pressure drops and friction factor are determined from CFD simulation throughout the flow direction by using both of resistance factors.

\section{Theory and Flow Field Governing Equations}

The porous media approach is generally used for the modeling of the problems encountered in the branches of science such as geology, nuclear energy and chemistry. It has also been used in the modeling of large surface area heat exchangers such as regenerator and automobile radiator $[17,18]$.

Since the distribution of pores is irregular in a natural porous environment, the distribution of flow magnitudes (velocity, pressure, etc.) on the pore scale (microscopic scale) is also irregular. However, in a typical experiment, flow magnitudes are measured in a macroscopic environment from many overlapping pores. The macroscopic data obtained from the experiments show similar flow properties to the regular variation of spatial-averaged macroscopic scale, depending on location and time. Therefore, this similarity enables the theoretical investigation of heat transfer and flow in porous media [19]. According to this approach, porous media flow and heat transfer can be modeled on a macroscopic scale by taking the spatial or statistical averages of conservation (mass, momentum and energy) equations.

The velocity of fluid flow in a porous medium is calculated by two approaches. The first; Darcy velocity, $\overline{\mathbf{v}}$ calculated according to the entire volume element (solid and fluid volume) forming the porous medium, the second; it is defined as the intrinsic velocity, $\overline{\mathbf{V}}$ (specific or leakage velocity) calculated according to the volume element occupied only by the fluid material. Both velocities are calculated by the volumetric average method. Darcy velocity directly linked to intrinsic velocity as follow,

$$
\overline{\mathbf{v}}=\varepsilon \overline{\mathbf{V}}
$$

where $\varepsilon$ is the porosity. By neglecting external forces of the compressible flow, for a porous medium, the continuity equation is expressed as

$$
\varepsilon \frac{\partial \overline{\rho_{f}}}{\partial t}+\tilde{\mathrm{N}} \cdot\left(\varepsilon \overline{\rho_{f} \mathbf{V}}\right)=0
$$

where $\overline{\rho_{f}}$ and $t$ denote mean fluid density and time respectively.

Vafai and Tien, Hsu and Cheng derived the general momentum equation for flow in a homogeneous and rigid isotropic porous medium [19, 20]. They arranged the microscopic equations into the macroscopicscaled equation form by taking the volume average on the representation volume element of the general equation. Macroscopic momentum equation of the porous medium stated as

$\rho_{f}\left[\frac{1}{\varepsilon} \frac{\partial \bar{v}}{\partial t}+\frac{1}{\varepsilon^{2}}(\bar{v} \cdot \nabla) \bar{v}\right]=-\nabla \overline{\rho_{f}}+$
$\tilde{\mu} \nabla^{2} \bar{v}-\frac{\mu}{\kappa} \bar{v}-\frac{c_{F}}{\kappa} \rho_{f}|\bar{v}| \bar{v}+\rho_{f} g$

The term on the left side of the last equation indicates momentum transport. The terms on the right side the $\mathrm{Eq}$ (3) from the left to right present pressure drop, viscous forces, viscous drag force, drag force, and body forces respectively. The symbols of $\tilde{\mu}, \mu, c_{F}$ and $\kappa$ given in Equation 3, denote effective viscosity, dynamic viscosity, dimensionless form-drag constant and intrinsic permeability respectively.

In ANSYS Fluent Software, the governing equation of the porous media flow are based on Forchheimer's law. The Forchheimer's flow model is an empirical equation resulting from experimental studies and is used to model the flow of high speed porous media with laminar regime [20]. The general Forchheimer's equation is stated as

$\nabla p=-\frac{\mu}{\kappa} \bar{v}-\frac{c_{F}}{\kappa} \rho_{f}|\bar{v}| \bar{v}$

In ANSYS Fluent theory guide, the last equation (4) is introduced as

$\frac{|\Delta p|}{L}=\frac{1}{\alpha} \mu v_{\infty}+\frac{C_{2}}{2} \rho v_{\infty}^{2}$

where $\frac{1}{\alpha}$ is the viscous inertial resistance factor and $C_{2}$ is the inertial resistance factor. In Eq. (5). The first term is the viscous loss and the second term is the inertial loss.

In this study, friction factor is calculated with the equation of Darcy-Weisbach via pressure drop calculated from the CFD simulation. The friction factor is calculated from

$f=\frac{D_{h}}{L} \frac{\Delta p}{\rho_{f} v_{h}^{2} / 2}$

where $D_{h}$ and $v_{h}$ denote hydraulic diameter and 
flow velocity respectively of porous media. The hydraulic diameter is expressed as

$D_{h}=\frac{\varepsilon d}{1-\varepsilon}$

where $d$ indicates wire diameter. Velocity $v_{h}$ is defined as follows

$v_{h}=\frac{Q}{\varepsilon A}$

where $Q$ and $\varepsilon A$ indicate flow rate and effective open area respectively [16]. The Reynolds number is calculated from

$R e_{h}=\frac{D_{h} v_{h}}{v}$

where $v$ is kinematic viscosity of the fluid.

\section{Validation of Viscous and Inertial Resistance Factors}

To examine the flow in a regenerator with porous media approach, CFD solver requires some constants such as viscous resistance and inertial resistance factors. Experimental data are needed to conduct to determine viscous and inertial resistance factors. As seen on Eq. (4) pressure drop is the function of inlet velocity. In this study, the resistance factors were determined using the experimental results of the study performed on a regenerator model by Yamashita and Hamaguchi [16]. The regenerator specifications to be used to validate the resistance factors are given in Table 1.

Table 1. Regenerator specifications and fluid type

\begin{tabular}{ll}
\hline Specifications & Descriptions \\
\hline Regenerator material & stainless steel \\
Porostiy $(\varepsilon)$ & $70 \%$ \\
Wire screen number & 150 \\
Wire diameter $(d)$ & $0.061 \mathrm{~mm}$ \\
Screen alignment & stacked wire \\
Length of the regenerator & $13.6 \mathrm{~mm}$ \\
Regenerator enterance diameter & $38 \mathrm{~mm}$ \\
Regenerator exit diameter & $34 \mathrm{~mm}$ \\
Fluid & air \\
\hline
\end{tabular}

Figure 1 indicates the experimental data of the regenerator that has specifications given in Table 1 and fitting data. By using the fitting equation constants, viscous and inertial resistance factors are determined as $10181.16 \times 10^{5}$ and 1030.78 respectively.

The regenerator, whose properties are given in Table 1, is modelled with design modeler of ANSYS software. The mesh structure of the solution domain is seen in Figure 2. To attain quick convergence and accurate results, mesh structure has to create considering the mesh quality parameters such as aspect ratio, skewness and orthogonal quality. In the simulation, the average of aspect ratio, skewness and orthogonal quality of solution domain mesh structure are $1.7843,0.10614$ and 0.9674 respectively.

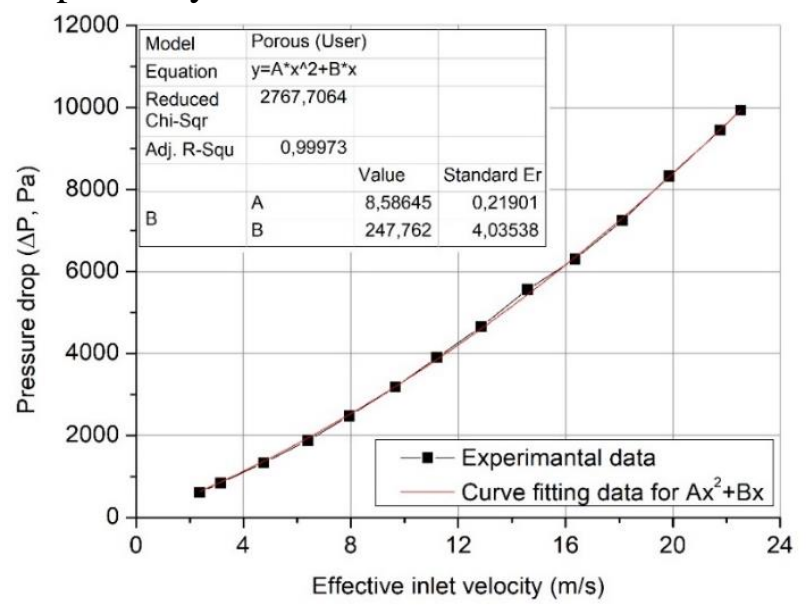

Figure 1. Experimental and curve fitting data

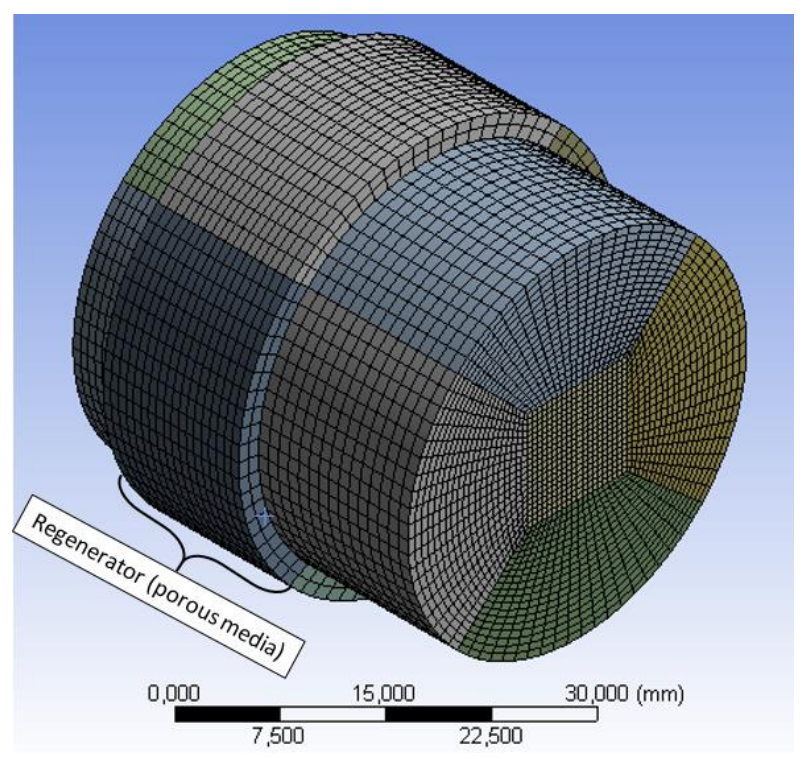

Figure 2. Regenerator model validated with CFD analysis

The simulation of the regenerator has been performed for $30 \mathrm{~L} / \mathrm{min}$ air flow rate using determined viscous and inertial resistance factors. In the experimental study conducted by Yamashita and Hamaguchi [16], the pressure drop is measured approximately as $600 \mathrm{~Pa}$ for $30 \mathrm{~L} / \mathrm{min}$ flow rate. The CFD calculations showed good agreement with experimental measurements for pressure drop and flow velocity profile. As a result, calculated pressure drop from the CFD analysis is $6 \%$ less than the experiment. As seen in Figure 3, both velocity profiles resemble each other. 


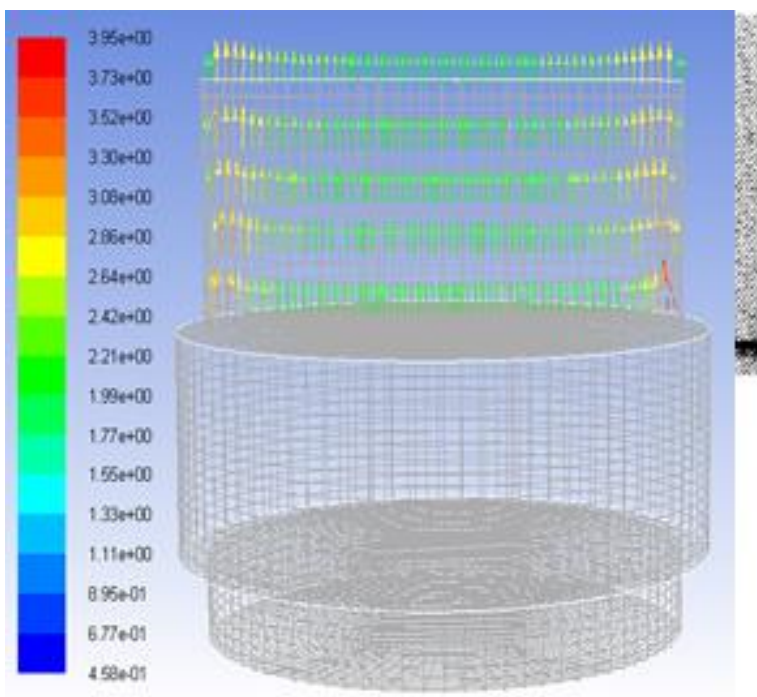

(a)

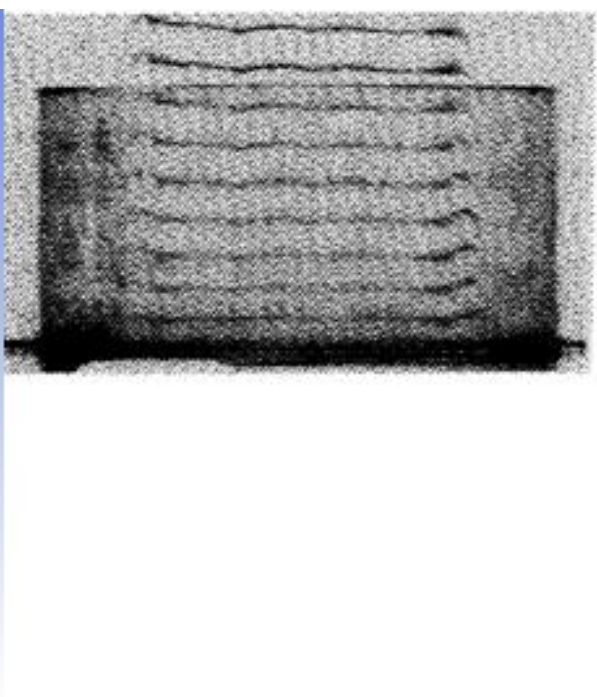

(b)

Figure 3. Velocity profile view at exit a) CFD result b) Experimental result [16]

\section{CFD Analysis of Proposed Stirling Engine Regenerator}

Figure 5 indicates location and dimensions of the proposed regenerator in the beta type Stirling engine. The regenerator shape is annular ring type. As seen in Figure 5a fluid flow interface at hot volume side of the regenerator is larger than the cold volume side. The regenerator is modelled considering this interface differences. Dimensions of the regenerator are given in Figure 5b.
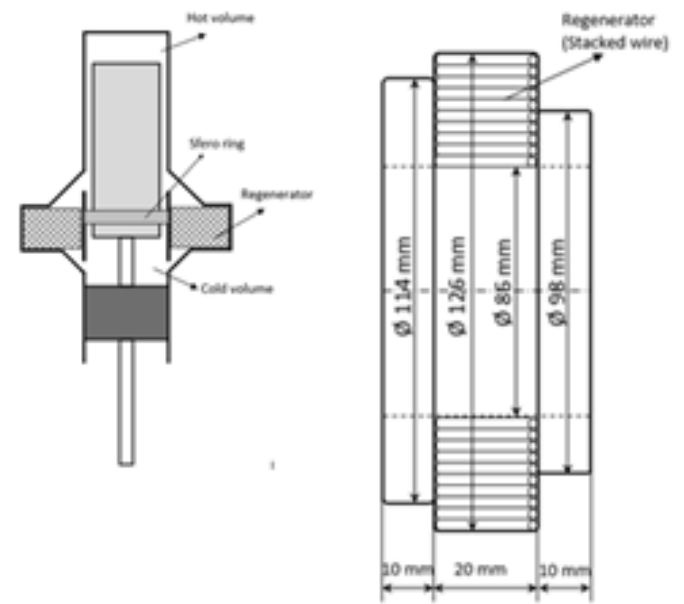

(b)

(a)

Figure 5. Proposed regenerator a) Location of the regenerator b) Regenerator dimensions

To ensure mesh independence of simulation results, the number of elements of the solution domain has been optimized for $4 \mathrm{~L} / \mathrm{s}$ fluid flow rate by considering mesh quality metrics. The average mesh quality values of aspect ratio, skewness, orthogonal quality and mesh grow rate are $1.6939,3.8272 \mathrm{e}-002,0.99735$ and 1.2 respectively. As seen in Figure 6 the value of the pressure loss is almost the same for the number of 180000 elements and above. All simulations are carried out using the 180000 elements in order to save solution time.

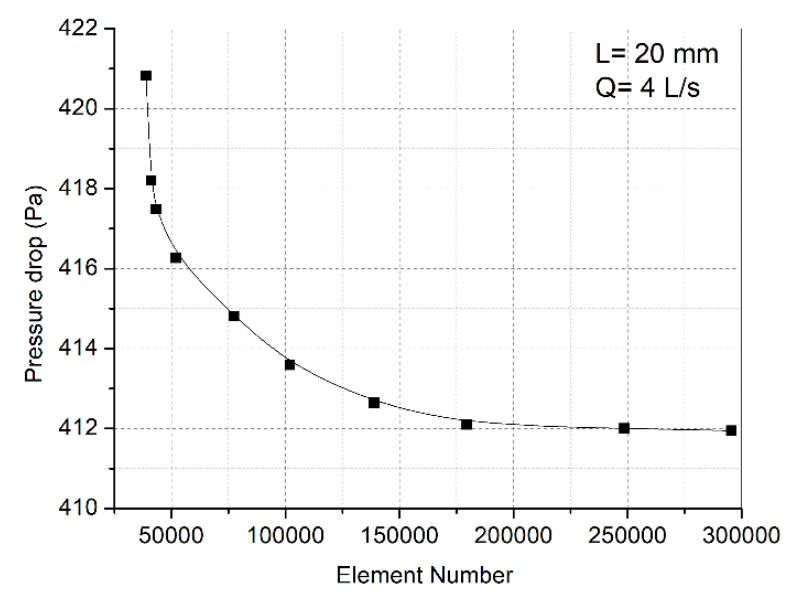

Figure 6. Mesh independency

In Figure 7, the variations of pressure drop and friction factor are presented for flow rate ranging from $4 \mathrm{~L} / \mathrm{s}$ to $200 \mathrm{~L} / \mathrm{s}$. Pressure drop values are taken from simulation results directly. Friction factor is calculated via Equation (5). For proposed regenerator hydraulic diameter with porous media $D_{h}$ and effective open area $\varepsilon A$ are calculated as $0.000412 \mathrm{~m}$ and $4.66 \times 10^{-3} \mathrm{~m}^{2}$ respectively by using Equation (6) and (7). While increasing the flow rate, the pressure drop is almost linearly increased and friction factor is reduced exponentially. After $60 \mathrm{~L} / \mathrm{s}$ flow rate, the friction drop is decelerating reduced because of increasing kinetic energy (proportional to velocity squared). 


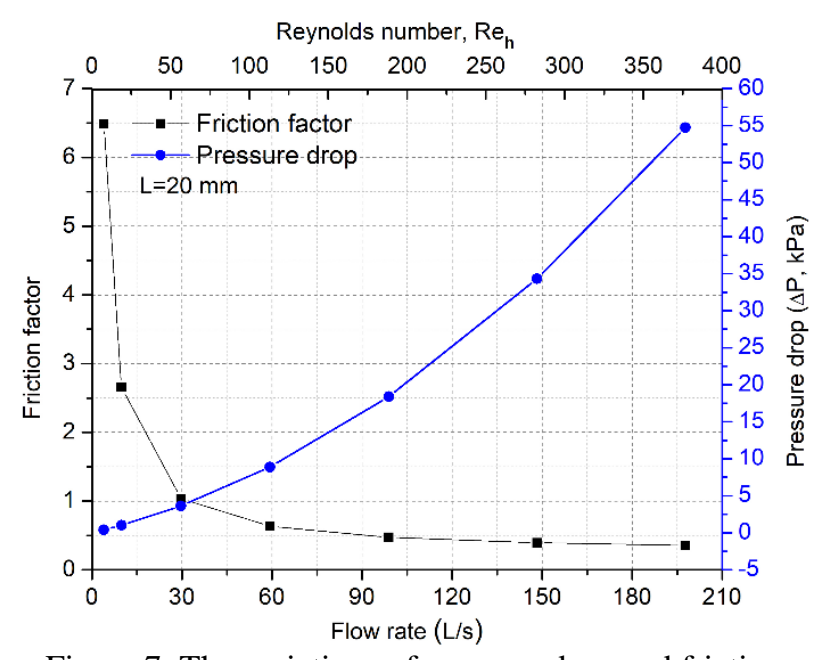

Figure 7 . The variations of pressure drop and friction factor

An exponential equation has been created by interpolating the calculated friction factor data depending on the flow rate given in Figure 7. This correlation equation is stated as

$$
\begin{aligned}
& f=14.502-12.61\left(1-e^{-\frac{Q}{4.109}}\right)- \\
& 1.528\left(1-e^{-\frac{Q}{35.56}}\right)
\end{aligned}
$$

where Q denotes the flow rate. Friction factor correlation depend on Reynolds number is expressed as

$$
f=50.6168 / R e_{h}+0.09443 R e_{h}^{0.15498}
$$

Provided that the porous media properties given in Table 1 remain the same, pressure drop and friction factor in different sizes of regenerators can be calculated with the Equation 10 or 11 . The last two equations provide to directly calculate the pressure drop of the regenerator in numerically modelled and simulated Stirling engines with codes such as Fortran, Matlab, $\mathrm{C}++$, and etc.

\section{Conclusions}

In the present study, the variations of pressure drop and friction factor of a proposed Stirling engine regenerator is determined with porous media approach via CFD software namely ANSYS Fluent. Flow properties in porous media obtained from published experimental data are used in the simulation and found to be good agreement between CFD simulation and experimental results. Viscous and inertial resistance factors were determined as $10181.16 \times 10^{5}$ and 1030.78 respecitvely for use in CFD analysis based on porous media. A friction correlation equation based on flow rate are created from simulation results to be used in numerically analysis of Stirling engine performance. More realistic results can be obtained by adding the correlation equations to the third order approach namely nodal analysis.

\section{References}

1. D. Beck, and D. G. Wilson, "Gas-turbine regenerators", Springer Science \& Business Media, 2012

2. K. Yanaga, "Experimental and Numerical Study of the Stirling Engine Robust Foil Regenerator", Graduate Theses, Dissertations, and Problem Reports, 7401, 2019. 3. M. Tanaka, I. Yamashita, F. Chisaka, "Flow and heat transfer characteristics of Stirling engine regenerator in oscillating flow", Trans. Japan Soc. Mech. Eng., Ser. B, 55, 24782485, 1989.

4. [4] P. Puech, V. Tishkova, "Thermodynamic analysis of a Stirling engine including regenerator dead volume", Renewable Energy, 36(2), 872-878, 2011.

5. S. K. Andersen, H. Carlsen, P. G. Thomsen, "Numerical study on optimal Stirling engine regenerator matrix designs taking into account the effects of matrix temperature oscillations", Energy Conversion and Management, 47(7-8), 894-908, 2006.

6. R. Gheith, F. Aloui and S. B. Nasrallah, "Determination of adequate regenerator for a Gamma-type Stirling engine", Applied Energy, 139, 272-280, 2015.

7. Z. S. Yuan, "Oscillatory flow and heat transfer in a Stirling engine regenerator", Doctoral dissertation, Case Western Reserve University, 1993.

8. M. C. Shin, J. A. and B. H. Kang, "Performance characteristics of a regenerative heat exchanger depending on its porous structure", Korean J. of Air-Conditioning and Refrigeration Engineering, 24 (6), 487-496, 2012 .

9. M. Arab, and M. Majidi, "Experimental and numerical study of porosity gradient in a Stirling engine regenerator" (No. 2017-010148), SAE Technical Paper, 2017.

10. W. L. Chen, K. L. Wong, H. E. Chen, "An experimental study on the performance of the moving regenerator for a $\gamma$-type twin power piston Stirling engine", Energy Conversion and 
Management, 77, 118-128, 2014.

11. S. C. Costa, H. Barrutia, J. A. Esnaola, M. Tutar, "Numerical study of the heat transfer in wound woven wire matrix of a Stirling regenerator", Energy Conversion and Management, 79, 255-264, 2014.

12. S. C. Costa, M. Tutar, I. Barreno, J. A. Esnaola, H. Barrutia, D. García, M. A. Gonzalez, J. I. Prieto, "Experimental and numerical flow investigation of Stirling engine regenerator", Energy, 72, 800-812, 2014.

13. B. G. Kim, W. S. Park, B. G. Koo, S. Park, "Effects of regenerator structure on performance of free piston Stirling engine (FPSE)", Journal of Mechanical Science and Technology, 32(9), 4473-4484, 2018.

14. M. A. Mohammadi, and A.Jafarian, "CFD simulation to investigate hydrodynamics of oscillating flow in a beta-type Stirling engine", Energy, 153, 287-300, 2018.

15. D. Gedeon, and J. G. Wood, "Oscillating-flow regenerator test rig: hardware and theory with derived correlations for screens and felts", NASA CR-198442, 6-13, 1996.

16. Yamashita, and K. Hamaguchi, "Effect of entrance and exit areas on the pressure drop and velocity distribution in regenerator matrix", JSME International Journal Series B Fluids and Thermal Engineering, 42(3), 498-505, 1999.

17. Y. Su, and J. H. Davidson, "Modeling approaches to natural convection in porous media", United States of America: Springer, 2015.

18. B. Çetin, K. G. Güler, and M. H. Aksel, "Computational modelling of vehicle radiators using prous medium approach. In Heat Exchanger Design, Experiment and Simulation", Chapter 11, INTECH, 2017.

19. D. A. Nield, and A. Bejan, "Convection in porous media", Volume 3. Springer, New York, 2006.

20. Vafai, K. Handbook of porous media. CRC Press, 2015. 